\title{
Sentinel Listeriosis Surveillance in Selected Hospitals, China, 2013-2017
}

\section{Weiwei Li, Li Bai, Xiaochen Ma, Xiuli Zhang, Xinpeng Li, Xiaorong Yang, Jennifer Y. Huang, Séamus Fanning, Yunchang Guo}

During 2013-2017, a total of 211 cases of listeriosis were reported by 64 sentinel hospitals in China to a national foodborne disease surveillance network. The average case-fatality rate was $31.2 \%$ for perinatal cases and $16.4 \%$ for nonperinatal cases. Sequence types 87 and 8 were the most prevalent types.

$\mathrm{L}$ isteriosis is caused by the gram-positive bacterium Listeria monocytogenes, which is ubiquitous in the environment and a foodborne pathogen of importance to public health. Listeriosis occurs sporadically and mainly in highrisk groups, such as pregnant women, neonates, and immunocompromised and elderly persons (1). Although listeriosis occurs rarely in humans, it has a high case-fatality rate of $20 \%-50 \%(2)$. Nearly all reported listeriosis cases are transmitted to humans via food (3), and L. monocytogenes can grow at refrigeration temperatures, which makes it particularly challenging to control (4).

In China, surveillance of L. monocytogenes in food products was launched in 2000 (5); however, as yet, listeriosis is not a notifiable disease in China. The National Foodborne Disease Surveillance Plan was implemented in $2011(6,7)$. Human listeriosis surveillance was included as a special pilot project in 2013. We provide an overview of the listeriosis sentinel surveillance data for the period 2013-2017. We summarize the demographic and clinical characteristics of patients with listeriosis and analyze the prevalent sequence types (STs) of all identified isolates.

Author affiliations: China National Center for Food Safety Risk Assessment, Beijing, China (W. Li, L. Bai, Y. Guo); Beijing Center for Disease Control and Prevention, Beijing (X. Ma); Henan Provincial Center for Disease Control and Prevention, Zhengzhou, China (X. Zhang); Shandong Provincial Center for Disease Control and Prevention, Jinan, China (X. Li); Sichuan Provincial Center for Disease Control and Prevention, Chengdu, China (X. Yang); Centers for Disease Control and Prevention, Atlanta, Georgia, USA (J.Y. Huang); University College Dublin, Dublin, Ireland (S. Fanning)

DOI: https://doi.org/10.3201/eid2512.180892

\section{The Study}

In 2013, listeriosis surveillance started in 6 selected provinces in China. The target was to detect whether human listeriosis existed in China and to determine illness and death rates for listeriosis. In 2017, this pilot surveillance had expanded to 12 provinces with the additional objectives to investigate high-risk factors and detect potential outbreaks (Figure). A total of 78 sentinel hospitals were selected using convenient sampling: 40 general hospitals, 28 maternity hospitals, and 10 children's hospitals.

We defined invasive listeriosis as the isolation of $L$. monocytogenes from a normally sterile site (e.g., blood or cerebrospinal fluid) or products of conception (e.g., placental or fetal tissue) (8). Pregnancy-associated patients were considered perinatal case-patients, including pregnant women, fetuses, or infants $\leq 28$ days of age; maternal-fetal infections were counted as a single case. We defined stillbirths and miscarriages as deaths, which were tallied in case-fatality rates. Pregnant women and neonates were the focused population groups, with immunocompromised and older adults also included. All demographic data, clinical manifestations, and laboratory tests were submitted to the China National Center for Food Safety Risk Assessment (CFSA) through the National Foodborne Disease Reporting System. All confirmed isolates were finally referred to CFSA for pulsed-field gel electrophoresis and wholegenome sequencing analysis through the National Molecular Tracing Network for Foodborne Disease Surveillance (TraNet).

During 2013-2017, a total of 211 listeriosis cases were diagnosed and reported by 64 sentinel hospitals, 138 $(65.4 \%)$ perinatal cases and $73(34.6 \%)$ nonperinatal cases. All case-patients were hospitalized; 55 deaths or fetal losses (case-fatality rate $26.1 \%$ ) were reported, and 43 (78.2\%) fatal cases occurred among fetuses and neonates. The average case-fatality rates were $31.2 \%(43 / 138)$ for perinatal and $16.4 \%$ (12/73) for nonperinatal cases. No maternal death was reported. Seventy-four (35.1\%) case-patients acquired listeriosis in the summer (June-August).

Of the 138 perinatal infections, the median age of the mother was 29 years (range 20-41 years), and the median gestational age was 32 weeks (range 8-40 weeks). Preterm labor ( $<37$ weeks gestational age) was reported in $63(45.7 \%)$ pregnant women with listeriosis. Clinical signs in pregnant women included intrauterine 


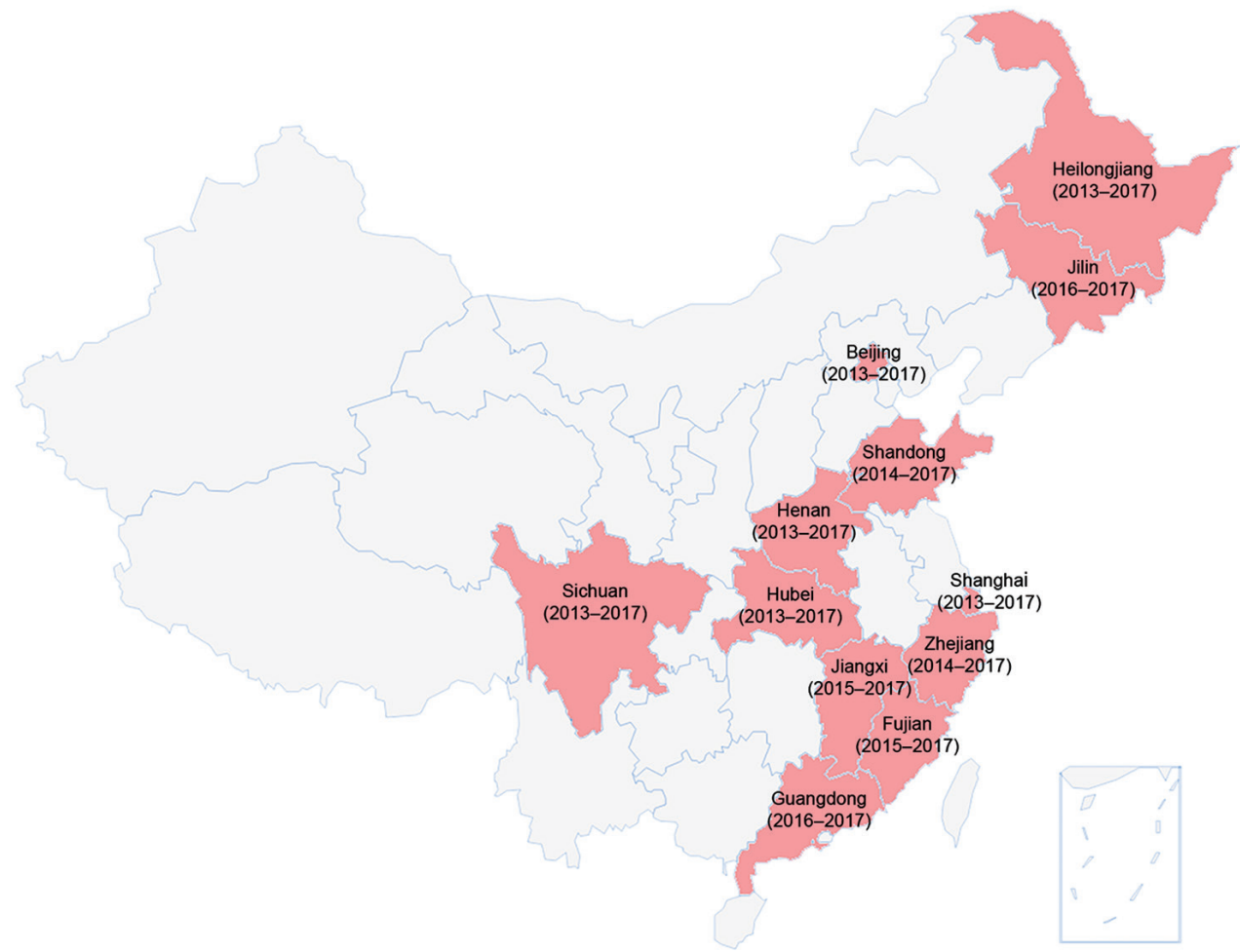

Figure. Geographic distribution of 12 selected provinces (red shading) included in human listeriosis surveillance, China, 2013-2017

infection, abortion, preterm labor, and influenza-like symptoms. Clinical manifestations and outcomes of infection in neonates included neonatal sepsis, asphyxia, pneumonia, meningitis, aspiration of amniotic fluid, meconium syndrome, and death.

Of the 73 nonperinatal infections, $45(61.6 \%)$ cases were bloodstream infections such as septicemia and bacteremia, $20(27.4 \%)$ were central nervous system infections, $6(8.2 \%)$ were acute gastroenteritis, and $2(2.7 \%)$ were focal infections. The median age of nonperinatal casepatients was 53 years (range 2 months-102 years); $22.9 \%$ were $>65$ years of age. The sex ratio was 1:1. Fifty-seven (78.1\%) patients had positive blood samples, 11 (15.1\%) had positive cerebrospinal fluid, and 15 (6.9\%) were positive in other specimens, such as pleural effusion, cystic liquid, bone marrow, and feces (Table 1). The all-cause immunosuppression rate was $28.8 \%$ (21/73 cases). We detected the following underlying immunosuppression conditions: hematologic malignancy, systemic lupus erythematosus, chronic obstructive pulmonary disease, chronic kidney disease, liver disease, organ tumor, lung transplantation, and tuberculosis.

Of the reported listeriosis cases, $28.9 \%$ (61/211) were followed up with epidemiologic investigation, and $18.0 \%$ (11/61) yielded positive results for L. monocytogenes in suspicious food, chopping boards, refrigerators, or kitchen sinks. However, the pulsed-field gel electrophoresis patterns were not identical to those of clinical isolates, and $>100$ allele differences were found by using the core genome multilocus sequence typing (MLST) profile of 1,748 loci (9). These results showed no links between food, environmental, and clinical isolates.

\begin{tabular}{lccc}
\hline \multicolumn{4}{l}{ Table 1. Demographic data of 211 listeriosis case-patients reported by 64 sentinel hospitals, by risk group, China, 2013-2017* } \\
\hline Characteristic & Pregnancy-associated, no. (\%) & Not pregnancy-associated, no. (\%) & Total, no. (\%) \\
\hline Total & $138(65.4)$ & $73(34.6)$ & $211(100.0)$ \\
\hline Sex & & & \\
F & $138(100.0)$ & $36(49.3)$ & $174(82.5)$ \\
M & 0 & 5 & 0 \\
Specimen source & & $57(78.1)$ & $143(67.8)$ \\
Blood & $86(62.3)$ & $11(15.1)$ & $19(9.0)$ \\
CSF & $8(5.8)$ & $5(6.9)$ & $5(2.4)$ \\
Othert & $\mathrm{NA}$ & $\mathrm{NA}$ & $44(20.9)$ \\
\hline Product of conception & $44(31.9)$ & $12(16.4)$ & $55(26.1)$ \\
\hline Death or fetal loss & $43(31.2)$ &
\end{tabular}

${ }^{*} \mathrm{CSF}$, cerebrospinal fluid; NA, nonapplicable.

†ncluding pleural effusion, cystic liquid, bone marrow, and feces. 
Table 2. Distribution of PCR serogroups and ST types identified among 108 isolates from listeriosis case-patients, by risk group, China, 2013-2017*

\begin{tabular}{lccc}
\hline Characteristic & Pregnancy-associated, no. (\%) & Not pregnancy-associated, no. (\%) & Total, no. (\%) \\
\hline Total & $76(70.4)$ & $32(29.6)$ & $108(100.0)$ \\
\hline PCR serogroups & & & \\
Ila & $30(39.5)$ & $15(46.9)$ & $45(41.7)$ \\
Ilb & $32(42.1)$ & $13(40.6)$ & $45(41.7)$ \\
IVb & $13(17.1)$ & $3(9.4)$ & $16(14.8)$ \\
Ilc & $1(1.3)$ & $1(3.1)$ & $2(1.9)$ \\
\hline MLST types & $14(18.4)$ & $3(9.4)$ & $17(15.7)$ \\
ST87 & $11(14.5)$ & $4(12.5)$ & $15(13.9)$ \\
ST8 & $4(5.3)$ & $4(12.5)$ & $8(7.4)$ \\
ST619 & $2(2.6)$ & $4(12.5)$ & $6(5.6)$ \\
ST155 & $45(59.2)$ & $17(53.1)$ & $62(57.4)$ \\
Other STs & & \\
\hline${ }^{*}$ MLST, multilocus sequencing typing; ST, sequence type. & & \\
\hline
\end{tabular}

A total of 116 isolates isolated during 2013-2016 were submitted to CFSA for whole-genome sequencing analysis: 108 from human listeriosis and 8 from the environment and suspicious food. The distribution of these 108 clinical L. monocytogenes clones was determined by MLST. A previous study reported that clonal complex (CC) 1, CC2, CC121, and CC155 were frequent clones in eastern Asia (10). We found that sequence type (ST) 87 (lineage I) and ST8 (lineage II) were the predominant STs; $15.7 \%$ of isolates were ST 87 and $13.9 \%$ were ST8. The prevalences of ST87 in clinical isolates (11) and in domestic food products were also reported previously (12). ST87 was seldom linked to human listeriosis in other countries; only 2 outbreaks (both in Spain) were associated with ST87 strains (13). The most common PCR serogroups were IIb and IIa (Table 2). A total of 89 different core genome MLST types were identified as groups that differ by up to 7 allelic mismatches among the clinical isolates.

\section{Conclusions}

Our study describes epidemiologic characteristics of listeriosis from sentinel surveillance in China. An estimated 1,662 cases of listeriosis occur each year in the United States (3); a detailed analysis should be expedited in China to estimate incidence. The Universal Two-Child Policy was proposed and passed in 2015, which likely will increase the number of pregnancies and births in China and might therefore increase the incidence of listeriosis.

This study has limitations (1). All cases came from sentinel hospitals but were not a complete picture of listeriosis occurrence because of the gradual increase of provinces included in surveillance (from 6 to 12 provinces), which meant the population served by selected hospitals could not be estimated accurately (2). All case-patients might be the most ill patients; cases might have been missed because those patients with milder illness might not go to the hospital and therefore will not be reflected in the data (3). The number of perinatal cases was nearly twice the number of nonperinatal cases, which cannot represent the actual illness and death rates because perinatal infection is given more attention in some sentinel hospitals (4). The case-fatality rates might be underestimated because all live-born infants, premature infants, and case-patients who did not complete follow-up surveillance were assumed to survive unless they were reported to have died.

In summary, health education and reasonable diet advice regarding listeriosis prevention should be provided to high-risk groups in China, and a focus on L. monocytogenes infection should be strengthened in hospitals. Moreover, L. monocytogenes is common in domestic food products in mainland China, especially in meat, poultry, seafood, and Chinese salad (14,15). An urgent need exists for improving surveillance of food and humans, exploring the mechanisms of pathogenesis, determining higher-risk foods, detecting potential outbreaks, and implementing control measures to protect vulnerable populations.

\section{Acknowledgments}

We thank all members in the sentinel hospitals and provincial Centers for Disease Control and Prevention for their enthusiastic participation in the human listeriosis surveillance program.

This research is funded by the National Key Research and Development Program of China (grant no. 2017YFC1601503).

\section{About the Author}

Mrs. Weiwei Li is an associate researcher in the Department of Risk Surveillance, China National Center for Food Safety Risk Assessment, Beijing, China. Her research interests include foodborne disease surveillance and molecular epidemiology of bacterial pathogens.

\section{References}

1. Lamont RF, Sobel J, Mazaki-Tovi S, Kusanovic JP, Vaisbuch E, Kim SK, et al. Listeriosis in human pregnancy: a systematic review. J Perinat Med. 2011;39:227-36. https://doi.org/10.1515/ jpm.2011.035 
2. Swaminathan B, Gerner-Smidt P. The epidemiology of human listeriosis. Microbes Infect. 2007;9:1236-43. https://doi.org/ 10.1016/j.micinf.2007.05.011

3. Scallan E, Hoekstra RM, Angulo FJ, Tauxe RV, Widdowson MA, Roy SL, et al. Foodborne illness acquired in the United States - major pathogens. Emerg Infect Dis. 2011;17:7-15. https://doi.org/10.3201/eid1701.P11101

4. de Noordhout CM, Devleesschauwer B, Angulo FJ, Verbeke G, Haagsma J, Kirk M, et al. The global burden of listeriosis: a systematic review and meta-analysis. Lancet Infect Dis. 2014;14:1073-82. https://doi.org/10.1016/ S1473-3099(14)70870-9

5. Pei X, Li N, Guo Y, Liu X, Yan L, Li Y, et al. Microbiological food safety surveillance in China. Int J Environ Res Public Health. 2015;12:10662-70. https://doi.org/10.3390/ ijerph120910662

6. Li W, Wu S, Fu P, Liu J, Han H, Bai L, et al. National molecular tracing network for foodborne disease surveillance in China. Food Control. 2018;88:28-32. https://doi.org/10.1016/ j.foodcont.2017.12.032

7. Liu J, Bai L, Li W, Han H, Fu P, Ma X, et al. Trends of foodborne diseases in China: lessons from laboratory-based surveillance since 2011. Front Med. 2018;12:48-57. https://doi.org/10.1007/ s11684-017-0608-6

8. Silk BJ, Date KA, Jackson KA, Pouillot R, Holt KG, Graves LM, et al. Invasive listeriosis in the Foodborne Diseases Active Surveillance Network (FoodNet), 2004-2009: further targeted prevention needed for higher-risk groups. Clin Infect Dis. 2012;54(Suppl 5):S396-404. https://doi.org/10.1093/ $\mathrm{cid} / \mathrm{cis} 268$

9. Moura A, Criscuolo A, Pouseele H, Maury MM, Leclercq A, Tarr C, et al. Whole genome-based population biology and epidemiological surveillance of Listeria monocytogenes. Nat Microbiol. 2016;2:16185. https://doi.org/10.1038/ nmicrobiol.2016.185

10. Chenal-Francisque V, Lopez J, Cantinelli T, Caro V, Tran C, Leclercq A, et al. Worldwide distribution of major clones of Listeria monocytogenes. Emerg Infect Dis. 2011;17:1110-2. https://doi.org/10.3201/eid/1706.101778

11. Wang Y, Jiao Y, Lan R, Xu X, Liu G, Wang X, et al. Characterization of Listeria monocytogenes isolated from human Listeriosis cases in China. Emerg Microbes Infect. 2015;4:e50. https://doi.org/10.1038/emi.2015.50

12. Wang Y, Zhao A, Zhu R, Lan R, Jin D, Cui Z, et al. Genetic diversity and molecular typing of Listeria monocytogenes in China. BMC Microbiol. 2012;12:119. https://doi.org/10.1186/14712180-12-119

13. Pérez-Trallero E, Zigorraga C, Artieda J, Alkorta M, Marimón JM. Two outbreaks of Listeria monocytogenes infection, Northern Spain. Emerg Infect Dis. 2014;20:2155-7. https://doi.org/10.3201/ eid2012.140993

14. Wu S, Wu Q, Zhang J, Chen M, Yan ZA, Hu H. Listeria monocytogenes prevalence and characteristics in retail raw foods in China. PLoS One. 2015;10:e0136682. https://doi.org/10.1371/ journal.pone. 0136682

15. Chen J, Zhang X, Mei L, Jiang L, Fang W. Prevalence of Listeria in Chinese food products from 13 provinces between 2000 and 2007 and virulence characterization of Listeria monocytogenes isolates. Foodborne Pathog Dis. 2009;6:7-14. https://doi.org/ $10.1089 /$ fpd.2008.0139

Address for correspondence: Yunchang Guo, China National Center for Food Safety Risk Assessment, No. 37, Bldg 2, Guangqulu, Chaoyang District, Beijing 100022, China; email: gych@cfsa.net.cn

\section{EID SPOTLIGHT TOPIC \\ Food Safety}

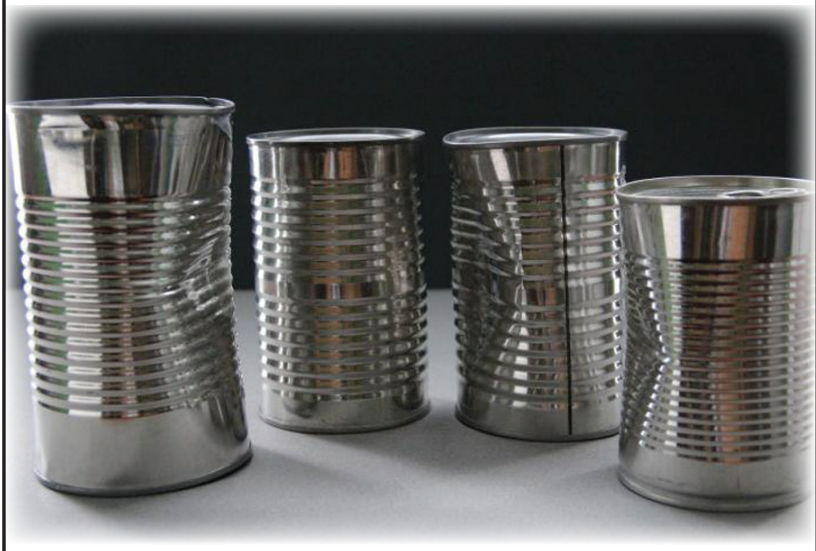

Foodborne illness (sometimes called "foodborne disease," "foodborne infection," or "food poisoning") is a common, costly-yet preventable-public health problem. Each year, 1 in 6 Americans gets sick by consuming contaminated foods or beverages. Many different disease-causing microbes, or pathogens, can contaminate foods, so there are many different foodborne infections. In addition, poisonous chemicals or other harmful substances can cause foodborne diseases if they are present in food.

\section{http://wwwnc.cdc.gov/eid/ page/food-safety-spotlight}

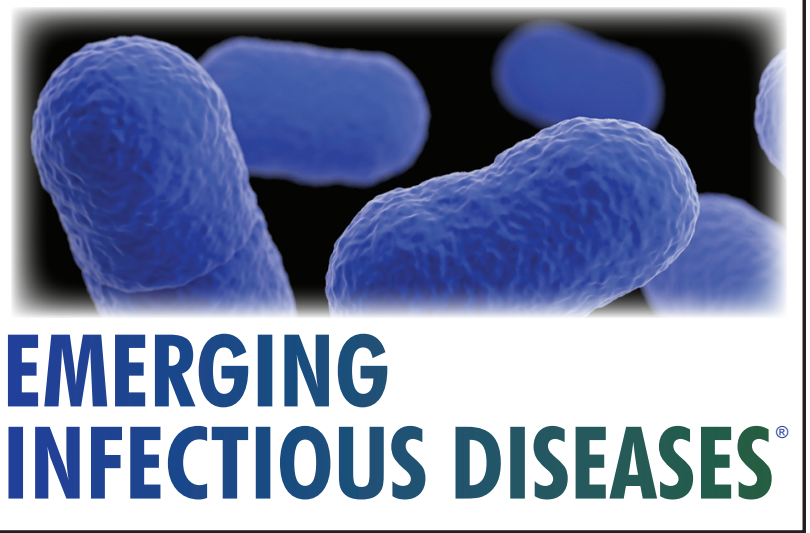

